\title{
Associations between dialysate interleukin- 6 and Tie-2 and peritoneal solute transport rate and outcomes for patients undergoing peritoneal dialysis: A prospective cohort study
}

\author{
Ying Hang ${ }^{1}$, Hao Yan' ${ }^{2}$, He Zhang ${ }^{3}$, \\ Zhenyuan $\mathrm{Li}^{4}$, Wei Fang ${ }^{5}$
}

\begin{abstract}
Objectives: We designed this prospective observational study to clarify the associations between dialysate IL-6, a marker of ongoing peritoneal inflammation, Tie2, an important factor in angiogenesis in the peritoneum, and a high peritoneal solute transport rate (PSTR) in patients undergoing peritoneal dialysis (PD) and to investigate their outcome predictive roles.

Methods: A total of 60 stable continuous ambulatory peritoneal dialysis (CAPD) patients from a single center in China were analyzed in this prospective study. We measured dialysate levels of IL-6 and Tie-2 using ELISAs. Our primary study endpoint was all-cause mortality with 10 years' follow-up.

Results: For the evaluation of PSTR, we used the Dialysis/Plasma creatinine (D/Pcr) ratio. We subdivided the patients into two groups for statistical evaluation: low and low average $\mathrm{D} / \mathrm{Pcr}(<0.64 ; \mathrm{L} / \mathrm{A})$, and high and high average D/Pcr $(\geq 0.65 ; \mathrm{H} / \mathrm{A})$ transporters. The mean levels of dialysates IL-6 $(21.71 \pm 8.88 \mathrm{pg} /$ $\mathrm{mL}$ ) and Tie-2 $(1.23 \pm 0.43 \mathrm{ng} / \mathrm{mL})$ were significantly higher in the $\mathrm{H} / \mathrm{A}$ (high and high average, group than those in the L/A group $(13.94 \pm 5.43 \mathrm{pg} / \mathrm{mL}, \mathrm{p}<0.001$ and $0.95 \pm 0.43 \mathrm{ng} / \mathrm{mL}, \mathrm{p}=0.019$; respectively). Moreover, IL- 6 and Tie-2 were positively correlated with D/Pcr $(r=0.366, p=0.004$ and $r=0.402, p=0.001$; respectively). Both dialysates IL- 6 and Tie- 2 were independent determinants of a high peritoneal solute transport rate. After follow-up for $42.65 \pm 18.08$ months, 30 patients $(50.0 \%)$ had died. An increased $\mathrm{D} / \mathrm{Pcr}$ increased the risk of all-cause mortality in patients with CAPD $(p=0.018)$, but the dialysates IL- 6 and Tie2 were not independent predictors of all-cause mortality $(p>0.05)$.

Conclusion: Our results suggest that patients undergoing CAPD have a high peritoneal solute transport status with local peritoneal inflammation and angiogenesis. Increased D/Pcr is a relative risk factor for mortality and technique failure in patients undergoing CAPD.
\end{abstract}

KEYWORDS: Angiogenesis, IL-6, Tie-2, High peritoneal solute transport rate, Peritoneal dialysis.

doi: https://doi.org/10.12669/pjms.37.4.4328

How to cite this:

Hang Y, Yan H, Zhang H, Li Z, Fang W. Associations between dialysate interleukin-6 and Tie-2 and peritoneal solute transport rate and outcomes for patients undergoing peritoneal dialysis: A prospective cohort study. Pak J Med Sci. 2021;37(4):1104-1110. doi: https://doi.org/10.12669/pjms.37.4.4328

This is an Open Access article distributed under the terms of the Creative Commons Attribution License (http://creativecommons.org/licenses/by/3.0), which permits unrestricted use, distribution, and reproduction in any medium, provided the original work is properly cited.

Correspondence:

Dr. Wei Fang,

Department of Nephrology,

Renji Hospital,

School of Medicine,

Shanghai Jiao Tong University,

Shanghai, China.

E-mail: fangwei_sh@126.com

* Received for Publication:

* Revision Received:

* Revision Accepted: *
February 24, 2021

March 18, 2021

April 2, 2021

\section{INTRODUCTION}

Peritoneal dialysis (PD) is a well-established renal replacement therapy for patients with end-stage renal disease (ESRD). ${ }^{1}$ Long-term preservation of peritoneal membrane function is a prerequisite for successful PDs. The standard peritoneal equilibration test (PET) is used to estimate the peritoneal small-solute transport rate. ${ }^{2}$ Patients undergoing PD can be classified into high $(\mathrm{H})$, high average (HA), low average (LA), and low (L) PET 
transporter types. The morphology and function of the peritoneal membrane changes during long-term continuous ambulatory peritoneal dialysis (CAPD) treatment, particularly in patients with high peritoneal solute transport rates (PSTRs) for small solutes. ${ }^{3}$ Angiogenesis, vasculopathy, and submesothelial fibrosis are typical histomorphological alterations of the peritoneal membrane ${ }^{4}$ with predisposing factors including long-term exposure to bio-incompatible dialysis fluid, recurrent peritonitis, and uremia. High concentrations of glucose in the dialysis fluid, glucose degradation products (GDPs), and advanced glycation endproducts (AGEs) induce inflammation, fibrosis, and angiogenesis via different pathways. ${ }^{5}$ The peritoneal permeability increases gradually with the duration of the PD treatment, while the peritoneal ultrafiltration function and volume decrease ${ }^{6,7} \mathrm{~A}$ high peritoneal permeability increases the risk of mortality and technical failure, and ultrafiltration failure has been associated with a high small-solute transport rate, as evidenced by a high D/Pcr. ${ }^{8,9}$

Interleukin-6 (IL-6) is a pleiotropic cytokine with roles in multiple pathological and physiological processes and a central regulator of inflammatory processes. Local intraperitoneal IL-6 production is a sign of an intraperitoneal inflammatory state. ${ }^{10}$

Tie-2, a transmembrane tyrosine kinase receptor for Ang ligands crucial for angiogenesis, blood vessel maturation, and vascular endothelium integrity. ${ }^{11}$ Serum Tie2 levels have been associated with different pathologies. Thus, the Ang/Tie-2 system is a primary regulator of angiogenesis and is involved in inflammation. ${ }^{12}$

We hypothesized that intraperitoneal inflammation and angiogenesis are interrelated and associated with a high peritoneal solute transport status in patients undergoing PD. In this study, we analyzed the intraperitoneal IL- 6 and Tie- 2 levels in 60 patients undergoing stable CAPD treatment with glucose-based solutions. Moreover, we assessed the association between intraperitoneal IL-6 and Tie-2, and their possible correlation to the PSTR during stable CAPDs.

\section{METHODS}

We performed this prospective observational study with data from a single center in China. We recruited all the study participants between July and December of 2007 and prospectively followed them to the end of the study 10 years later (January 31, 2018). All procedures followed in this study were in accordance with the ethical standards of the responsible committee on human experimentation (institutional and national) and with the Helsinki Declaration of 1975. The Human Research Ethics Committee of Renji Hospital, School of Medicine, Shanghai Jiao Tong University approved the study protocol (Approval Number 2016101K). All participants gave their written informed consent.

The 60 patients on CAPD represented all of the clinically stable patients treated in the center and were regularly followed up in the Dialysis Unit of Renji Hospital affiliated to Shanghai Jiao Tong University School of Medicine. This population consisted of 29 men and 31 women with a mean age of $57.9 \pm 13.9$ years (range, $18-83$ years), treated with CAPD for an average of $34.2 \pm 29.1$ months (3149.6 months). Table-I lists the ESRD causes for the patients in our study.

On the night before the first dialysis fluid drain for the study, all the patients underwent a dialysis exchange using a $2.5 \%$ glucose solution (Dianeal ${ }^{\circledR}$, Baxter, USA) and recorded its exact time. ${ }^{13}$ We collected $10-\mathrm{mL}$ samples of dialysate and immediately stored them at $-70^{\circ} \mathrm{C}$. We then initiated a standard fast peritoneal equilibrium test (PET) ${ }^{14}$ Dialysate samples for glucose and creatinine were collected at times 0 and $240 \mathrm{~min}$.

For the evaluation of PSTR we used the D/Pcr ratio. ${ }^{14}$ We divided the patients according to the PET classification into high (D/Pcr $\geq 0.81 ; n=5)$, high average (D/Pcr 0.65-0.80; $n=16)$, low average (D/ Pcr 0.51-0.64; $n=22)$, and low (D/Pcr $\leq 0.50 ; n=17)$ transporters. In addition, we subdivided the patients into only two groups for statistical evaluation: low and low average D/Pcr $(<0.64 ; \mathrm{L} / \mathrm{A})$, and high and high average $\mathrm{D} / \mathrm{Pcr}(\geq 0.65 ; \mathrm{H} / \mathrm{A})$ transporters.

We determined the concentrations of cytokines in the dialysis effluent after the night dwell using the ELISA technique. All samples were run simultaneously and in duplicate for each mediator to avoid intra- and inter-assay variations. IL-6 and Tie- 2 levels were determined using Quantikine ${ }^{\circledR}$ kits (R\&D Systems Inc, USA). Both of the assays are considered highly specific for the cytokines, and we observed no significant cross-reactivity. The plates were read using a microplate reader (Bio-Rad, USA).

We followed up all the patients prospectively from the enrollment of the study until death, cessation of PD, transfer to other centers, or the end of the study (January 31, 2018). We evaluated death from all causes as the primary outcome, and limited the survival analysis to the first event.

Statistical analysis: We expressed results as mean values \pm standard deviations (SDs), and used 
Dialysate interleukin-6 and Tie-2

Table-I: Contrast of general data between L/A and H/A groups (means \pm SD or medians with ranges).

\begin{tabular}{|c|c|c|c|}
\hline Group & $L / A$ group $(n=39)$ & H/A group $(n=21)$ & $p$-value \\
\hline Age (years) & $56.8 \pm 14.3$ & $59.8 \pm 13.3$ & 0.44 \\
\hline Gender (men/women) & $16 / 23$ & $13 / 8$ & 0.12 \\
\hline Diabetes Mellitus & $17.9 \%$ & $14.3 \%$ & 0.66 \\
\hline Peritonitis (episodes/months) & 0.0059 & 0.0051 & 0.63 \\
\hline PD duration (month) & $27.97(3-80)$ & $30.03(3-149.6)$ & 0.66 \\
\hline 4-h drained volume (mL) & $2326.15 \pm 142.12$ & $2276.67 \pm 124.07$ & 0.19 \\
\hline Over-night drained volume (mL) & $2249.74 \pm 286.83$ & $2036.19 \pm 293.44$ & 0.008 \\
\hline Ultrafiltration (mL/24h) & $550.00(-800-1650)$ & $940.00(-180-2080)$ & 0.02 \\
\hline e GFR $(\mathrm{mL} / \mathrm{min} / 1.73 \mathrm{~m} 2)$ & $1.74(0-8.10)$ & $1.72(0-6.00)$ & 1.00 \\
\hline residual UV (mL/24h) & $428.21(0-1300)$ & $533.33(0-2000)$ & 0.93 \\
\hline $\mathrm{D} / \mathrm{P} \mathrm{cr}$ & $0.51 \pm 0.07$ & $0.75 \pm 0.11$ & $<0.001$ \\
\hline $\mathrm{Kt} / \mathrm{Vt}$ & $2.00 \pm 0.40$ & $1.94 \pm 0.44$ & 0.59 \\
\hline $\mathrm{Kt} / \mathrm{V} \mathrm{p}$ & $1.66 \pm 0.32$ & $1.58 \pm 0.43$ & 0.42 \\
\hline $\mathrm{Kt} / \mathrm{V} \mathrm{r}$ & $0.37(0-1.41)$ & $0.36(0-1.29)$ & 0.96 \\
\hline $\operatorname{Ccr} \mathrm{t}(\mathrm{L} / \mathrm{w} / 1.73 \mathrm{~m} 2)$ & $58.47 \pm 19.82$ & $63.07 \pm 21.22$ & 0.41 \\
\hline Ccr p (L/w/1.73 m2) & $40.68 \pm 8.02$ & $44.63 \pm 10.03$ & 0.10 \\
\hline $\operatorname{Ccr} \mathrm{r}(\mathrm{L} / \mathrm{w} / 1.73 \mathrm{~m} 2)$ & $17.79(0-82.71)$ & $18.44(0-60.48)$ & 0.91 \\
\hline n PCR (g/kg/day) & $0.87 \pm 0.20$ & $0.89 \pm 0.13$ & 0.79 \\
\hline CRP (mg/L) & $14.06(3.10-32.80)$ & $9.38(3.12-15.00)$ & 0.12 \\
\hline Glucose (mmol/L) & $6.37 \pm 1.44$ & $6.43 \pm 1.60$ & 0.88 \\
\hline Albumin (mg/L) & $38.56 \pm 3.45$ & $35.89 \pm 3.58$ & 0.006 \\
\hline dialysate IL-6 (pg/mL) & $13.94 \pm 5.43$ & $21.71 \pm 8.88$ & 0.001 \\
\hline dialysate Tie-2 (ng/mL) & $0.95 \pm 0.43$ & $1.23 \pm 0.43$ & 0.019 \\
\hline
\end{tabular}

Values expressed as means \pm SD or medians with ranges (min-max). peritoneal dialysis (PD), estimated glomerular filtration rate (e GRF), residual urinary volume (UV), Dialysis/Plasma creatinine (D/Pcr), Dialyzer clearance of urea multiplied by dialysis time and normalized for urea distribution volume (Kt/V), Kt/V total (Kt/V t), Kt/V peritonaeum (Kt/V p), Kt/V residual renal (Kt/V r), Clearance of creatinine (Ccr), Ccr total (Ccr t), Ccr peritonaeum (Ccr p), Ccr Residual renal (Ccr r), normalized protein catabolic rate (n PCR), C-reactive protein (CRP).

medians and ranges for non-normally distributed parameters. To compare differences between two transport groups, we used unpaired Student $t$-tests. When normal distribution was not present, we applied a non-parametric analysis (MannWhitney test). To compare D/Pcr values with their respective cytokine levels, we applied a linear regression analysis. We generated cumulative survival curves using the Kaplan-Meier method. We also applied the Cox proportional hazards model to estimate the relative risks of all-cause mortality for different variables. We performed all statistical tests using the SPSS software version 25.0 for Windows (SPSS, USA).

\section{RESULTS}

The characteristics of the two transport groups are described in Table-I. We found no significant age, gender, dialysis age, or diabetes prevalence differences between the H/A and the L/A groups. We found similar values for serum C-reactive protein $(C R P)(p=0.12)$ and for peritonitis rates
$(\mathrm{P}=0.63)$ in both groups. The $\mathrm{D} / \mathrm{Pcr}$ values were $0.51 \pm 0.07$ in the $\mathrm{L} / \mathrm{A}$ group and $0.75 \pm 0.11$ in the $\mathrm{H} / \mathrm{A}$ group, with the $\mathrm{D} / \mathrm{Pcr}$ in the $\mathrm{H} / \mathrm{A}$ group being significantly higher $(p<0.001)$. The dialysis adequacy and residual renal function in the two groups were also similar. However, the overnight drained volume in the $\mathrm{H} / \mathrm{A}$ group was significantly lower than that in the $\mathrm{L} / \mathrm{A}$ group $(\mathrm{p}=0.008)$. The net ultrafiltration in the $\mathrm{H} / \mathrm{A}$ group was significantly lower than that in the L/A group $(p=0.02)$. Finally, the serum albumin was significantly lower in the $\mathrm{H} / \mathrm{A}$ group than in the L/A group $(\mathrm{p}=0.006)$.

Dialysate cytokines and peritoneal transport rate of solutes: The concentration of IL-6 in the dialysate was significantly higher in the $\mathrm{H} / \mathrm{A}$ group than in the L/A group $(\mathrm{p}=0.001)$ (Fig.1A). The dialysate concentration of IL-6 was significantly correlated with the D/Pcr ( $r=0.366, p=0.004)$ (Fig.2A).

Meanwhile, the concentration of Tie-2 in dialysate was significantly higher in the $\mathrm{H} / \mathrm{A}$ group than in the L/A group ( $p=0.019$ ) (Fig.1B). The dialysate concentration of Tie-2 was significantly correlated 


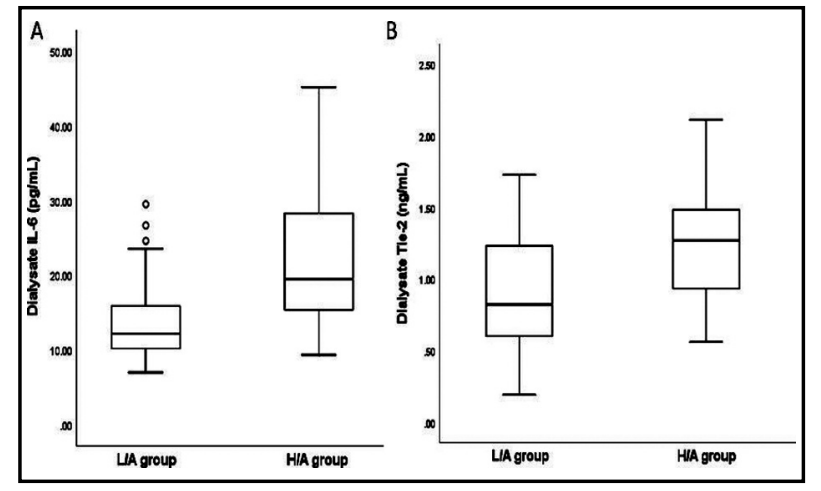

Fig.1: Comparison of dialysate IL-6 (A) and Tie-2 (B) concentrations between $\mathrm{L} / \mathrm{A}$ and $\mathrm{H} / \mathrm{A}$ groups.

Box plot representation: $75 \%$ percentile, $25 \%$ percentile, median, and maximum and minimum values.

with both the D/Pcr ( $r=0.402, \mathrm{p}=0.001$; Fig.2B) and the blood glucose level ( $\mathrm{r}=0.316, \mathrm{p}=0.014$; Fig.3). On the other hand, the dialysate concentration of Tie-2 was negatively correlated with the overnight drained volume $(r=-0.332, p=0.009)$. We found no correlation between the dialysate concentrations of IL-6 and Tie-2 ( $\mathrm{r}=0.023, \mathrm{p}=0.862)$.

Multiple regression analysis of $\mathrm{D} / \mathrm{Pcr}$ influencing factors: We carried out a multivariate linear regression analysis with age, dialysis age, BMI (body mass index), blood glucose, albumin, CRP, dialysate concentration of IL-6, and the dialysate concentration of Tie- 2 as the independent variables, and with the D/Pcr value as the dependent variable. Our results show that the dialysate concentrations of IL-6 $(\beta=0.007, p=0.026)$ and Tie$2(\beta=0.162, p=0.006)$ were both independent risk factors for D/Pcr (Table-II).

Kaplan-Meier estimates of overall survival probability: After a mean follow-up of $42.65 \pm 18.08$ months, 30 patients $(50.0 \%)$ had died and $21(35.0 \%)$ had 21 continued CAPD; two patients $(3.3 \%)$ had undergone kidney transplantation, $6(10.0 \%)$ had been switched to permanent hemodialysis therapy, and one patient $(1.7 \%)$ had transferred to another

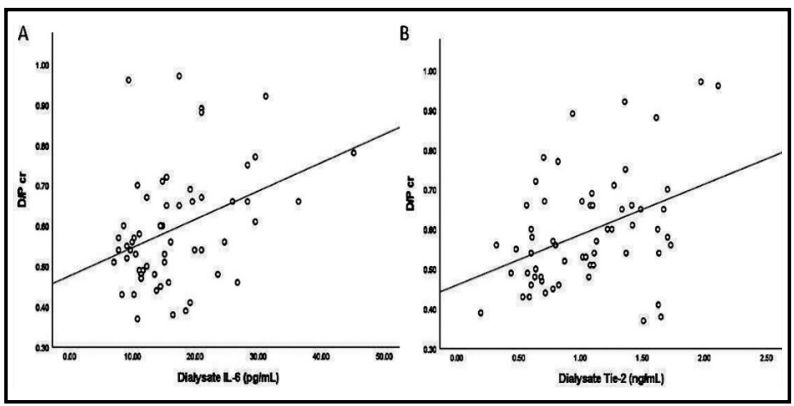

Fig.2: Correlations of dialysate IL-6 (A) and Tie-2 (B) concentrations with D/Pcr levels.

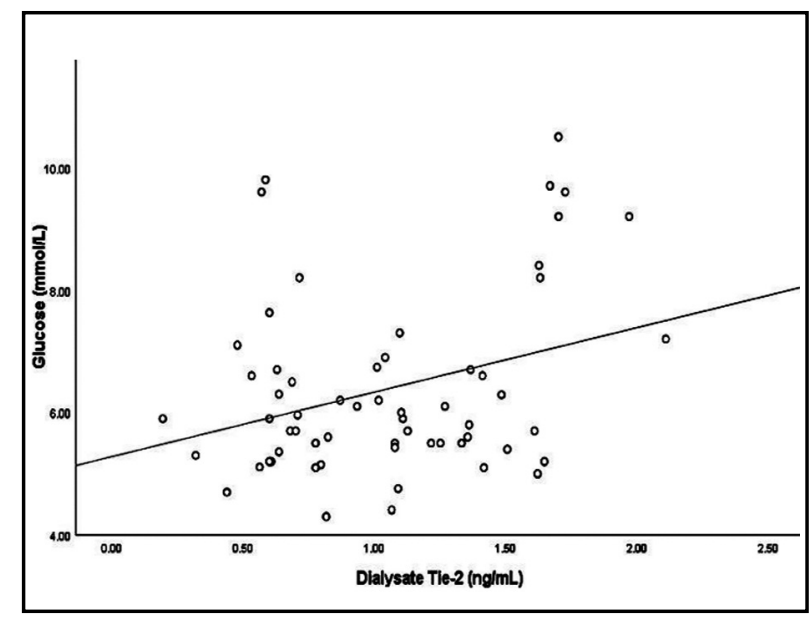

Fig.3: Correlation of dialysate Tie-2 concentration with serum glucose levels.

center. According to the Kaplan-Meier survival analysis, the all-cause mortality was significantly higher in the L/A group than in the H/A group $(p=0.018$; Fig.4), while other factors between the two groups of D/Pcr were similar (all p>0.05).

\section{DISCUSSION}

The patients on PD and with high peritoneal permeability had an increased small-solute PSTR; the peritoneal osmotic pressure gradient disappeared rapidly, resulting in a reduced fluid clearance rate. A high PSTR reflects the large effective peritoneal surface area resulting from a large number of perfused peritoneal capillaries engaged in the diffusion of the solute, and the increased permeability of the peritoneal

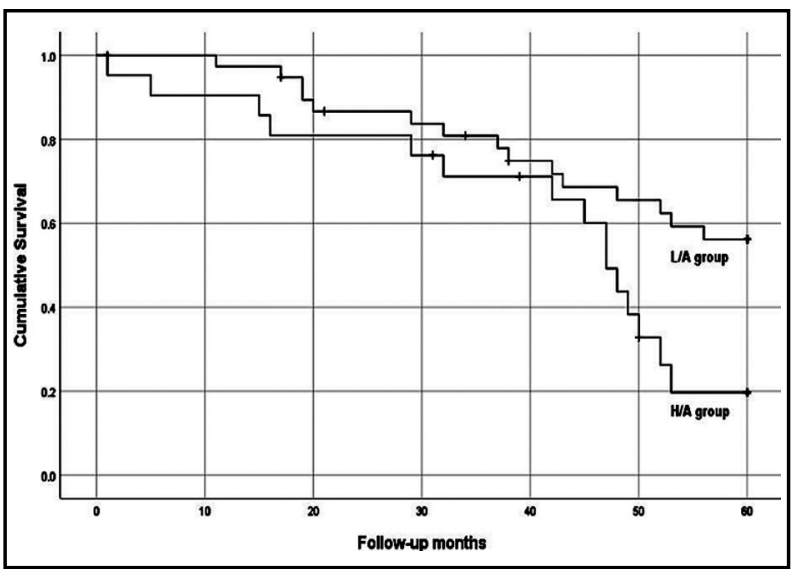

Fig.4: Kaplan-Meier estimates of overall survival probability of patients stratified according to D/Pcr. Low or low average transporters (L/A group; D/Pcr $<0.64,39$ patients) and high or high average transporters (H/A group; D/Pcr $\geq 0.65$, 21 patients). All-Cause mortality (Log rank=5.629, $\mathrm{p}=0.018$ ). 
Table-II: Multivariate regression analysis for D/Pcr.

\begin{tabular}{lcc}
\hline \multicolumn{3}{c}{$\mathrm{D} / \mathrm{Pcr}$} \\
\hline & Standardized coefficient $\beta$ & $p$-value \\
\hline age & 0.000 & 0.478 \\
PD duration & 0.000 & 0.956 \\
BMI & -0.003 & 0.811 \\
Glu & 0.006 & 0.767 \\
Alb & 0.001 & 0.897 \\
CRP & 0.002 & 0.916 \\
dialysate IL-6 & 0.007 & 0.026 \\
dialysate Tie-2 & 0.162 & 0.006 \\
\hline
\end{tabular}

vasculature. The most common functional alteration during long-term CAPD is an increased peritoneal small-solute transport rate. ${ }^{15}$ Studies have shown that a high peritoneal permeability is an important risk factor for mortality and technical failure in patients undergoing long-term PD. ${ }^{8,9}$

We found that patients with higher PSTR had higher levels of dialysate IL-6 and Tie-2, indicating these are closely associated with a small-solute PSTR. Moreover, we found that dialysate concentrations of IL-6 and Tie-2 were independent risk factors of $\mathrm{D} /$ Pcr. These results suggest a link between inflammation, angiogenesis, and the small-solute PSTR. However, we found no association between dialysate concentrations of IL- 6 and Tie- 2 .

The acute phase inflammatory response factor IL-6 is secreted by different cells in response to diverse stimuli, it induces the production of acute phase proteins and regulates the temporal switch from acute to chronic inflammation. ${ }^{16}$ Dialysate concentrations of IL-6 reflect an intraperitoneal inflammatory state with cytokine levels being higher in the dialysate than in plasma, showing further increases during peritonitis. ${ }^{11}$ Inflammatory states in patients undergoing CAPD can be due to the use of bio-incompatible dialysis fluids that may induce peritoneal mesothelial cells, macrophages, and endothelial cells to produce of IL-6. ${ }^{17}$ Moreover, high transporter patients undergoing PD have increased levels of dialysate IL-6 that are associated with the PSTR. ${ }^{13,18,19}$ Longitudinal studies have also correlated the dialysate IL-6 levels with baseline D/Pcrs. ${ }^{20}$ Also, both IL-6 in plasma and dialysate correlate with dialysis adequacy in patients undergoing CAPD. ${ }^{21}$

In this study, the level of dialysate IL-6 was significantly higher in the H/A group than in the L/A group. Moreover, the dialysate concentration of IL-6 had a positive correlation with the D/Pcr. Consistent with the results of a previous study, ours also suggested a close link between a high PSTR and local peritoneal inflammation, and we found that the high peritoneal transport patients presented a more severe local intraperitoneal inflammation state than the others.

IL-6 has been detected in the dialysate of patients lacking signs of systemic inflammation. ${ }^{22}$ In this study, dialysate concentrations of IL-6 did not correlate with the serum CRP concentrations. Local peritoneal production of IL-6 may reflect a chronic inflammatory state of the peritoneum, apart from systemic inflammation. Also, we found a discrepancy in serum albumin levels between the H/A and L/A groups. Hypoalbuminemia has been associated with high peritoneal transport patients undergoing PD. ${ }^{23}$ IL-6 may increase peritoneal leakage of proteins, which may reflect endothelial dysfunction. In addition, significantly prolonged inflammation contributes to a poor nutritional status and higher mortality. ${ }^{24}$ However, hypoalbuminemia in patients undergoing PD is considered a multifactorial condition with dialysisrelated and non-dialysis-related factors. Tie- 2 can be detected in the blood vessels of normal tissues..$^{25}$

The principal water and solute barriers in PD are the blood capillary endothelium and the interstitial matrix. The peritoneal permeability increases in patients undergoing PD and, eventually, leads to ultrafiltration failure. Peritoneal biopsies in patients undergoing PD have shown that extensive angiogenesis is associated with peritoneal treatment duration. ${ }^{26}$ Angiogenesis is thought to result in an increased effective surface exchange area, whereas lymphangiogenesis leads to raised lymphatic absorption rates. The expanded vascular network results in a decrease in the glucose-driven osmotic pressure of the PD fluid leading to ultrafiltration loss. In addition, vascular wall thickening and increased permeability cause an increase in small solute transport and result in a reduction time for exchanging waste products. ${ }^{27,28}$ A cartilage oligomeric matrix protein (COMP)-Ang-1 treatment may significantly increase the level of phosphorylation of the Tie-2 receptor in uremic peritoneal dialysis rats, suggesting that preservation of the Ang-1/Tie2 signal may alleviate peritoneal membrane injuries and improve the peritoneal transport function. ${ }^{29}$ Peritoneal angiogenesis may be the main factor increasing the peritoneal solute transport. ${ }^{30}$ Markers, such as Tie-2, could be used to obtain information on "peritoneal angiogenesis". We showed that the dialysate Tie-2 level in the H/A group was significantly higher than that in the L/A group, suggest- 
ing that a high PSTR may be due to increased peritoneal angiogenesis. Moreover, we also found that the dialysate Tie-2 levels were positively correlated with the D/Pcr ratio. The dialysate concentration of Tie-2 was an independent risk factor for a high $\mathrm{D} / \mathrm{Pcr}$, suggesting that patients undergoing PD who are high small solute peritoneal transporters may present increased peritoneal angiogenesis; we found angiogenesis to be an independent risk factor for peritoneal permeability.

Interestingly, we also found that the dialysate Tie-2 level was positively correlated with the blood glucose level. Glucose is a proinflammatory agent with additional profibrotic effects. A high concentration of glucose in the dialysate may promote angiogenesis and fibrosis of the peritoneal membrane through a stimulation of the polyol pathway. ${ }^{31}$ GDPs produce more AGE products than glucose. GDPs and AGEs are pro-inflammatory factors that can be induced by VEGF-mediated angiogenesis. ${ }^{4}$ We confirmed results from previous studies showing that in addition to a high glucose concentration in the dialysis fluid, the peripheral blood glucose concentration may also promote peritoneal angiogenesis.

Studies have demonstrated an intimate connection between a high transport status and poor outcomes. ${ }^{8,32,33}$ Meta-analyses have indicated that the relative risks for mortality and technique failure increase by 1.15 and 1.18 (respectively) for every 0.1 increase in the dialysate over plasma ratio for D/Pcr. ${ }^{7}$ The mortalities of the LA, HA, and high transport groups increased by $21.9 \%, 45.7 \%$ and $77.3 \%$, respectively, as compared to that of the low transport group. We found a significant increase in all-cause mortality between the L/A group and the H/A group with a 60-month followup confirming that an increased $\mathrm{D} / \mathrm{Pcr}$ is a relative risk factor for mortality and technique failure in patients undergoing CAPD. Cardiovascular disease (CVD) events were the leading cause of death in our patients, followed by cerebrovascular events. Studies have reported positive associations between circulating Tie-2 and cardiovascular risk factors. ${ }^{34,35}$ Meanwhile, chronic inflammation is a remarkable risk factor for atherosclerotic disease particularly in patients undergoing CAPD. ${ }^{36}$ Our results show that the patients in the H/A group had higher levels of dialysate IL- 6 and Tie- 2 than patients in the L/A group, but we did not test circulating IL-6 and Tie-2 levels in these patients. Therefore, we cannot confirm that dialysate IL- 6 and Tie- 2 are relative risk factors for mortality and technique failure in patients undergoing CAPD. We can only speculate the existence of an association between intraperitoneal inflammation, angiogenesis, and the outcome of patients undergoing CAPD.

Limitations: First of all, we only performed single-time-point measurements of dialysate concentrations of IL- 6 and Tie- 2 that may not reflect changes over time or time-averaged exposures. Second, the number of cases included in this study is relatively small. another well-designed and extensive study will be required to confirm our findings.

\section{CONCLUSION}

In summary, our results suggest that patients undergoing CAPD have a high PSTR with local peritoneal inflammation and angiogenesis. The dialysate concentrations of IL-6 and Tie-2 both seem to be independent risk factors for peritoneal permeability. In our patients, peritoneal local inflammation and angiogenesis were both involved in the peritoneal permeability changes observed. Increased D/Pcr was a relative risk for mortality and technique failure in these patients.

Competing interests: The authors declare that they have no competing interests.

Funding: No funding was received.

Availability of data and materials: The datasets used and/or analyzed during the current study are available from the corresponding author on reasonable request.

Ethical approval: The Human Research Ethics Committee of Renji Hospital, School of Medicine, Shanghai Jiao Tong University approved the study protocol (Approval No. 2016101K).

\section{REFERENCES}

1. Xu XD, Han X, Yang Y, Li X. Comparative study on the efficacy of peritoneal dialysis and hemodialysis in patients with end-stage diabetic nephropathy. Pak J Med Sci. 2020;36(7):1484-1489. https:/ / doi.org/10.12669/pjms.36.7.2901

2. Twardowski ZJ, Nolph KO, Khanna R, Prowant BF, Ryan LP, Moore HL, et al. Peritoneal Equilibration Test. Perit Dial Int. 1987;7(3):138-148.

3. Schilte MN, Celie JWAM, Wee PM Ter, Beelen RHJ, van den Born J. Factors contributing to peritoneal tissue remodeling in peritoneal dialysis. Perit Dial Int. 2009;29(6):605-617. doi: 10.1177/089686080902900604

4. Williams JD, Craig KJ, Topley N, Williams GT. Peritoneal dialysis: changes to the structure of the peritoneal membrane and potential for biocompatible solutions. Kidney Int Suppl. 2003;(84):S158-S161. doi: 10.1046/j.1523-1755.63.s84.46.x

5. Fusshoeller A. Histomorphological and functional changes of the peritoneal membrane during long-term peritoneal dialysis. Pediatr Nephrol. 2008;23(1):19-25. doi: 10.1007/s00467-007-0541-z 
6. Davies SJ. Longitudinal relationship between solute transport and ultrafiltration capacity in peritoneal dialysis patients. Kidney Int. 2004;66(6):2437-2445. doi: 10.1111/j.1523-1755.2004.66021.x

7. Unal A, Hayri Sipahioglu M, Kocyigit I, Elmali F, Tokgoz B, Oymak $O$. Does body mass index affect survival and technique failure in patients undergoing peritoneal dialysis? Pak J Med Sci. 2014;30(1):414. doi: 10.12669 / pjms.301.3807

8. Rumpsfeld M, McDonald SP, Johnson DW. Higher peritoneal transport status is associated with higher mortality and technique failure in the Australian and New Zealand peritoneal dialysis patient populations. J Am Soc Nephrol. 2006;17(1):271-278. doi: 10.1681/ASN.2005050566

9. Brimble KS, Walker M, Margetts PJ, Kundhal KK, Rabbat CG. Metaanalysis: peritoneal membrane transport, mortality, and technique failure in peritoneal dialysis. J Am Soc Nephrol. 2006;17(9):25912598. doi: 10.1681/ASN.200603019

10. Lai KN, Lai KB, Lam CW, Chan TM, Li FK, Leung JC. Changes of cytokine profiles during peritonitis in patients on continuous ambulatory peritoneal dialysis. Am J Kidney Dis. 2000;35(4):644-652. doi: 10.1016/S0272-6386(00)70011-4

11. Yancopoulos GD, Davis S, Gale NW, Rudge JS, Wiegan SJ, Holash J. Vascular-specific growth factors and blood vessel formation. Nature. 2000;407(6801):242-248. doi: 10.1038/35025215

12. Saharinen P, Leppanen V-M, Alitalo K. SnapShot: Angiopoietins and Their Functions. Cell. 2017;171(3):724-724.e1. doi: 10.1016/j. cell.2017.10.009

13. Pecoits-Filho R, Araujo MRT, Lindholm B, Stenvinkel P, Abensur H, Romao JE, et al. Plasma and dialysate IL- 6 and VEGF concentrations are associated with high peritoneal solute transport rate. Nephrol Dial Transplant. 2002;17(8):1480-1486. doi: 10.1093/ndt/17.8.1480

14. Twardowski ZJ. PET--a simpler approach for determining prescriptions for adequate dialysis therapy. Adv Perit Dial. 1990;6:186-191.

15. Hung KY, Huang JW, Tsai TJ, Chen WY. Natural changes in peritoneal equilibration test results in continuous ambulatory peritoneal dialysis patients: a retrospective, seven-year cohort survey. Artif Organs. 2000;24(4):261-264. doi:10.1046/j.15251594.2000.06478.x

16. Hurst SM, Wilkinson TS, McLoughlin RM, Jones S, Horiuchi S, Yamamoto N, et al. Il-6 and its soluble receptor orchestrate a temporal switch in the pattern of leukocyte recruitment seen during acute inflammation. Immunity. 2001;14(6):705-714. doi: 10.1016/ S1074-7613(01)00151-0

17. Witowski J, Jorres A, Coles GA, Williams JD, Topley N. Superinduction of IL-6 synthesis in human peritoneal mesothelial cells is related to the induction and stabilization of IL- 6 mRNA Kidney Int. 1996;50(4):1212-1223. doi:10.1038/ki.1996.430

18. Yang $X$, Zhang $H$, Hang $Y$, Yan $H$, Lin A, Huang J, et al. Intraperitoneal interleukin-6 levels predict peritoneal solute transport rate: a prospective cohort study. Am J Nephrol. 2014;39(6):459-465. doi: $10.1159 / 000362622$

19. Rodrigues A, Martins M, Santos MJ, Fonseca I, Oliveira JC, Cabrita A, et al. Evaluation of effluent markers cancer antigen 125, vascular endothelial growth factor, and interleukin-6: relationship with peritoneal transport. Adv Perit Dial. 2004;20:8-12. doi: $10.1159 / 000099332$

20. Oh K-H, Jung JY, Yoon MO, Song A, Lee $\mathrm{H}$, Ro $\mathrm{H}$, et al. Intraperitoneal interleukin-6 system is a potent determinant of the baseline peritoneal solute transport in incident peritoneal dialysis patients. Nephrol Dial Transplant. 2010;25(5):1639-1646. doi: $10.1093 /$ ndt/gfp670

21. Boonyarittipong $M$, Kurathong S, Trakarnvanich T. Interleukin-6, procalcitonin, and vascular endothelial growth factor in plasma and dialysate correlate with dialysis adequacy in continuous ambulatory peritoneal dialysis patients. Clin Nephrol. 2019;92(5):273-275. doi: 10.5414/CN109559

22. Findley CM, Cudmore MJ, Ahmed A, Kontos CD. VEGF induces Tie2 shedding via a phosphoinositide 3-kinase/Akt dependent pathway to modulate Tie2 signaling. Arterioscler Thromb Vasc Biol. 2007;27(12):2619-2626. doi: 10.1161/ATVBAHA.107.150482

23. Margetts PJ, McMullin JP, Rabbat CG, Churchill DN. Peritoneal membrane transport and hypoalbuminemia: cause or effect? Perit Dial Int. 2000;20(1):14-18. doi: 10.1177/089686080002000104

24. Lam MF, Leung JCK, Lo WK, Tam S, Chong M, Lui SL, et al. Hyperleptinaemia and chronic inflammation after peritonitis predicts poor nutritional status and mortality in patients on peritoneal dialysis. Nephrol Dial Transplant. 2007;22(5):1445-1450. doi: $10.1093 / \mathrm{ndt} / \mathrm{gfl} 788$
25. Wong AL, Haroon ZA, Werner S, Dewhirst MW, Greenberg CS, Peters KG. Tie2 expression and phosphorylation in angiogenic and quiescent adult tissues. Circ Res. 1997;81(4):567-574. doi: 10.1161/01. res.81.4.567

26. Mateijsen MA, van der Wal AC, Hendriks PM, Zweers MM, Mulder J, Struijk DG, et al. Vascular and interstitial changes in the peritoneum of CAPD patients with peritoneal sclerosis. Perit Dial Int. 1999;19(6):517-525. doi: 10.1177/089686089901900605

27. Michels WM, Zweers MM, Smit W, Korevaar J, Struijk DG, van Westrhenen R, et al. Does lymphatic absorption change with the duration of peritoneal dialysis? Perit Dial Int. 2004;24(4):347-352. doi: $10.1177 / 089686080402400409$

28. Lai KN, Tang SCW, Leung JCK. Mediators of inflammation and fibrosis. Perit Dial Int. 2007;27(Suppl 2):S65-S71. doi: 10.1177/089686080702702s12

29. Shi Y, Xiong Y, Lei Y, Li Z, Yan H, Yuan J, et al. Protective effect of COMP-angiopoietin-1 on peritoneal vascular permeability and peritoneal transport function in uremic peritoneal dialysis rats. Am J Transl Res. 2019;11(9):5932-5943.

30. Margetts PJ, Kolb M, Yu L, Hoff CM, Gauldie J. A chronic inflammatory infusion model of peritoneal dialysis in rats. Perit Dial Int. 2001;21(Suppl-3):S368-S372. doi: 10.1177/089686080102103S70

31. van Westrhenen $R$, Aten I, Aberra M, Dragt CAM, Deira G, Krediet RT. Effects of inhibition of the polyol pathway during chronic peritoneal exposure to a dialysis solution. Perit Dial Int. 2005;25(Suppl 3):S18-S21. doi: 10.1177/089686080502503S05

32. Chung $\mathrm{SH}$, Heimburger $\mathrm{O}$, Lindholm B. Poor outcomes for fast transporters on PD: the rise and fall of a clinical concern. Semin Dial. 2008;21(1):7-10. doi: 10.1111/j.1525-139X.2007.00327.x

33. Janda K, Krzanowski M, Dumnicka P, Kusnierz-Cabala B, Miarka $\mathrm{P}$, Sułowicz W. Peritoneal solute transport rate as an independent risk factor for total and cardiovascular mortality in a population of peritoneal dialysis patients. Adv Perit Dial. 2014;30:15-20.

34. Lieb W, Zachariah JP, Xanthakis V, Safa R, Chen M-H, Sullivan LM, et al. Clinical and genetic correlates of circulating angiopoietin-2 and soluble Tie-2 in the community. Circ Cardiovasc Genet. 2010;3(3):300-306. doi: 10.1161/CIRCGENETICS.109.914556

35. Rasul S, Reiter MH, Ilhan A, Lampichler K, Wagner L, KautzkyWiller A. Circulating angiopoietin-2 and soluble Tie-2 in type 2 diabetes mellitus: a cross-sectional study. Cardiovasc Diabetol. 2011;10:55. doi: 10.1186/1475-2840-10-55

36. Wang AY-M, Lai K-N. Use of cardiac biomarkers in end-stage renal disease. J Am Soc Nephrol. 2008;19(9):1643-1652. doi: 10.1681/ ASN.2008010012

\section{Authors' contributions:}

YH and WF conceived, designed the study and are responsible for integrity of the study.

HZ and ZL collected the data.

HY performed the analysis.

YH was involved in the writing of the manuscript. All authors have read and approved the final manuscript.

Authors:

1. Ying Hang,

Department of Emergency,

2. Hao Yan,

Department of Nephrology,

3. He Zhang,

Department of Nephrology,

4. Zhenyuan $\mathrm{Li}$,

Department of Nephrology,

5. Wei Fang,

Department of Nephrology,

1-5: Renji Hospital, School of Medicine,

Shanghai Jiao Tong University, Shanghai, China. 